\title{
Características clínicas gingivales de pacientes portadores de prótesis fija tratados en una clínica dental docente
}

\section{Gingival clinical characteristics of patients with fixed prosthesis treated in a teaching dental clinic}

Rosa Raquel Huivín-Rodríguez ${ }^{1, a}$, Gian Marco Gonzáles-Pinedo ${ }^{2, a}$, Beatriz Del Carmen Chávez-Reátegui ${ }^{3, a, b, d}$, Jorge Enrique Manrique-Chávez ${ }^{4, a, c, e}$

\section{RESUMEN}

Objetivo: Identificar las características clínicas gingivales de las piezas portadoras de prótesis fijas (PF) de pacientes que acudieron a la Clínica Dental Docente de la Universidad Peruana Cayetano Heredia, para ser atendidos por alumnos de pregrado, el año 2012. Material y métodos: Estudio de diseño retrospectivo con un universo conformado por historias clínicas de pacientes portadores de prótesis fijas. Resultados: De las 196 historias clínicas evaluadas, se encontraron: 250 prótesis fijas, las cuales tuvieron un total de 339 pilares, entre los tipos: coronas unitarias $(63,2 \%)$ y pilares de puente $(36,8 \%)$. Existió mayor prevalencia de pacientes de género masculino $(53,06 \%)$, con una edad media de 42.61 años. El análisis descriptivo univariado de los datos mostró mayor prevalencia de piezas pilares posterosuperior $(45,2 \%)$, gingivitis moderado $(51,6 \%)$, ausencia de retención de placa $(50,4 \%)$, ausencia de movilidad dentaria $(90,3 \%)$ y estado malo de la prótesis fija $(71,7 \%)$. El análisis bivariado, encontró relación estadísticamente significativa $(\mathrm{p}<0.05)$ entre las variables: tipo de prótesis - retención de placa, tipo de prótesis - movilidad, estado de prótesis - índice gingival y estado de prótesis - retención de placa. Por otro lado, no se encontró relación entre las variables: tipo de prótesis - índice gingival y género e índice gingival. Conclusiones: Se encontró relación entre el estado periodontal y la presencia de prótesis fija, lo cual se expresa a través en la alta prevalencia de gingivitis moderada y presencia de retención de placa en las piezas pilares de las prótesis fijas.

PALABRAS CLAVE: Dentadura parcial, dentadura parcial fija, periodoncia, gingivitis. (DeCS, BIREME)

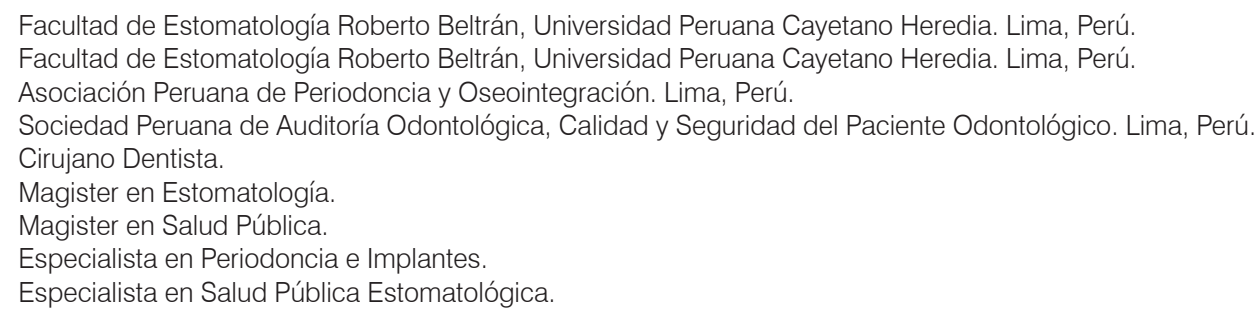




\section{SUMMARY}

Objective: Identify the clinical gingival characteristics of carriers tooth fixed prostheses of patients attending at the Teaching Dental Clinic (UPCH) in 2012. Methods: A retrospective design was conducted with a universe of clinical records of patients with fixed prosthesis. Results: Of the 196 clinical histories evaluated, we found: 250 fixed prostheses, which consisted of 339 pillars of fixed prostheses between types: single crowns (63.2\%) and bridge abutments (36.8\%). Higher prevalence of male patients $(53.06 \%)$ gender was found, with an average age of 42.61 years. In univariate descriptive analysis highest prevalence was found in the posterior parts (45.2\%), moderate gingival status (51.6\%), absence of plaque retention (50.4\%), absence of tooth mobility (90.3\%) and poor condition of fixed prostheses (71.7\%). In bivariate analysis, statistically significant relationship ( $p$ $<0.05)$ between the variables type of prosthesis-plaque-retaining, type of prosthesis-mobility, state prosthesisgingival index and state prosthesis-plaque-retaining was found; on the other hand was no relationship between the variables type of prosthesis-gingival index and gender-gingival index was found. Conclusions: There is a relationship between periodontal status and the presence of fixed prosthesis, which is expressed through the high prevalence of moderate gingivitis and plaque retention presence of abutment teeth for fixed prosthesis.

KEYWORDS: Denture partial, denture partial fixed, periodontics, gingivitis. (MeSH, NLM)

\section{INTRODUCCIÓN}

Se emplea la prótesis parcial fija para el reposicionamiento de piezas perdidas (puente), o individualmente para restaurar o corregir algún defecto en forma o malposición dentaria (coronas) (1).

El tejido dentario perdido debe ser sustituido con un material biocompatible con los tejidos gingivales, que no permita la formación de placa y que no contribuya a la inflamación gingival. Mediante restauraciones de metal-cerámica y libres de metal, se pueden reemplazar grandes áreas de estructura dentaria perdidas; al mismo tiempo, que se protege y preserva el remanente dentario (2).

La estructura de soporte de las piezas dentarias es el periodonto, y si éste se ve afectado y se genera su pérdida, conllevará a largo plazo a la pérdida de piezas dentarias. Es por ello que, se debe tener muy en consideración el cuidado del periodonto al momento de realizar cualquier tratamiento restaurador (3).

Diversos estudios $(4,5,6,7,8,9,10)$ han sido realizados para determinar si las restauraciones indirectas o prótesis fijas (puentes, coronas) provocan cambios en los tejidos periodontales, en mayor o menor medida, durante los procedimientos que se llevan a cabo para su confección.
Con el fin de conseguir resultados estéticos y funcionales a largo plazo, el clínico debe asegurarse que el tejido gingival esté sano antes de comenzar con la preparación definitiva del diente, así como al momento de cementar la prótesis fija, ya que un periodonto saludable reaccionará de manera predecible y positiva a la agresión inevitable de la intervención odontológica; en cambio en una situación de patología, la agresión empeorará las condiciones del tejido $(3,7,9)$.

El objetivo del presente estudio fue identificar las características clínicas gingivales de las piezas portadoras de prótesis fijas (PF) de pacientes que acudieron a la Clínica Dental Docente de la Universidad Peruana Cayetano Heredia, para ser atendidos por alumnos de pregrado, el año 2012.

\section{MATERIAL Y MÉTODOS}

Diseño de tipo retrospectivo, observacional, descriptivo y de corte transversal que estudio las características clínicas gingivales de pacientes portadores de prótesis parcial fija (PPF) que acudieron a la Clínica Dental de la Universidad Peruana Cayetano Heredia (UPCH) en el año 2012, para ser atendidos por alumnos de pregrado. El tamaño del universo estuvo conformado por 196 historias clínicas. 
Los criterios de inclusión fueron: pacientes recibidos por los alumnos de pregrado de la Facultad de Estomatología de la Universidad Peruana Cayetano Heredia, entre los 18 y 60 años de edad y portadores de PPF. Los criterios de exclusión fueron: pacientes con tratamiento ortodóncicos, pacientes con tratamientos médicos (últimos 6 meses), pacientes con tratamientos quirúrgicos, pacientes con enfermedades sistémicas y pacientes con diagnóstico de periodontitis o en tratamiento periodontal.

Se empleó una matriz de datos que contenían las siguientes variables: género, edad, tipo de prótesis, ubicación, estado gingival. Las variables: retención de placa y movilidad, se valorizaron en: no presenta y presenta; el estado de la prótesis fija, se valoró como: bueno (adaptado, sin fractura) y malo (desadaptado, fracturado). La información obtenida de los registros de historias clínicas fue sobre: Índice Gingival (Loe y Silness, 1963), Índice de Retención (Bjorby y Loe, 1967), Índice de Movilidad Dentaria (Miller, 1938) y situación de la prótesis (OMS, 1997).

Para el análisis descriptivo univariado y bivariado fueron utilizadas las pruebas estadísticas: t de Student, Chi cuadrado de Pearson y prueba exacta de Fisher. Se tomó en cuenta un valor de $\mathrm{p}<0,05 \mathrm{y}$ el intervalo de confianza del 95\% como el nivel de significancia estadística.

El protocolo de investigación fue revisado y aprobado por las autoridades de la Facultad de Estomatología Roberto Beltrán, el Departamento Académico de Clínica Estomatológica y por el Comité Institucional de Ética de la Universidad Peruana Cayetano Heredia.

\section{RESULTADOS}

Se evaluaron 196 historias clínicas encontrandose 250 prótesis fijas, que constaban de 339 pilares de prótesis fijas (coronas unitarias y pilares de puente). El universo estuvo conformado por $53,06 \%$ pacientes de género masculino $y$ un $46,94 \%$ de género femenino, con una edad media de 42,61 años de edad. Encontrándose un rango de mayor prevalencia de PPF fue entre 40 y 49 años de edad (33,16\%).

El análisis descriptivo univariado mostró que del total de pilares de prótesis fijas, el 63,2\% fueron coronas individuales y $36,8 \%$ pilares de puentes. Según su ubicación: $26 \%$ de PPF estuvieron en la zona anterosuperior, $45,2 \%$ en posterosuperior, $28 \%$ posteroinferior y $0,8 \%$ anteroinferior. Según el estado gingival se encontró: gingivitis leve en el $45,1 \%$, moderada en el $51,6 \%$ y severa en el 3,2\%. Respecto a retención de placa, se encontró: presencia de placa 49,6\% y ausencia de placa 50,4\%. Se encontró ausencia de movilidad en el 90,3\% de los casos y presencia de movilidad en sólo el 9,7\%. En lo que se refiere al estado de la PPF, se encontró: 28,3\% en buen estado y $71,7 \%$ en mal estado (Tabla 1 ).

Según el análisis descriptivo bivariado se encontró relación estadísticamente significativa $(\mathrm{p}<0.05)$ entre las siguientes variables: tipo de prótesis - retención de placa, tipo de prótesis - movilidad, estado de prótesis - índice gingival, estado de prótesis - retención de placa. Sin embargo, el estudio no encontró relación

Tabla 1. Características de los pacientes portadores de prótesis fija, que acudieron a la clínica dental docente de la UPCH.

\begin{tabular}{clcc}
\hline Variable & \multicolumn{1}{c}{ Categoría } & N & \% \\
\hline \multirow{2}{*}{ Género } & Femenino & 92 & 46,94 \\
& Masculino & 104 & 53,06 \\
Tipo de & Corona individual & 158 & 63,2 \\
prótesis & Puente & 92 & 36,8 \\
& & & \\
& Antero superior & 65 & 26,0 \\
Ubicación de & Antero inferior & 2 & 0,8 \\
prótesis & Posterior superior & 113 & 45,2 \\
& Posterior inferior & 70 & 28,0 \\
& & & \\
Estado de & Bueno & 96 & 28,3 \\
prótesis fija & Malo & 243 & 71,7 \\
& & 153 & 45,13 \\
& Leve (0-1) & 175 & 51,62 \\
Estado gingival & Moderado (1.1-2) & 11 & 3,25 \\
& Severo (2.1-3) & & \\
& & 171 & 50,4 \\
Retención de & No presenta & 168 & 49,6 \\
placa & Presenta & & \\
& & 306 & 90,3 \\
Movilidad & No presenta & 9,7 \\
\hline
\end{tabular}


estadísticamente significativa $(\mathrm{p}>0.05)$ entre las siguientes variables: tipo de prótesis - índice gingival, género e índice gingival.

\section{DISCUSIÓN}

Los resultados obtenidos en el presente estudio difieren de los estudios de Bustos y Oyanader (4), Valderhaug y Karlsen (5), Anweigi y col. (6), que muestran una mayor prevalencia del género femenino; encontrándose en este estudio que el 53,06\% fue del género masculino. En cuanto a la edad, se obtuvo una media de 42,61 años, mostrando como rango más frecuente las edades entre 40 y 49 años.

El tipo de prótesis fija encontrada con mayor frecuencia fue: corona individual $(63,2 \%)$ y puentes $(36,8 \%)$. Estos resultados muestran a las coronas individuales como los tratamientos restauradores más frecuentes, coincidiendo con los estudios de Näpänkangas y col. (7), Zitzmann y col. (8), Valderhaug y Karlsen (5).

La ubicación más frecuente de las prótesis fijas fue en la zona posterosuperior $(45,2 \%)$, seguida de la posteroinferior (28\%), anterosuperior $(26 \%)$, y anteroinferior $(0,8 \%)$. Estos resultados son similares a los encontrados por Anweigi y col. (6), quienes observaron $40 \%$ de ellas en el sector posterior, $24 \%$ en el sector anterior y $36 \%$ combinación de ambos. Estudios como los de Silness (9) y Valderhaug y Karlsen (5), muestran mayor frecuencia de coronas y PPF en el maxilar superior, afirmando que las piezas superiores reciben estos tratamientos más temprano que las inferiores, además muestran que las piezas molares y premolares tienen mayor tendencia a desarrollar caries o enfermedad periodontal, y por ello requieren ser reemplazadas o restauradas más tempranamente. Esto puede deberse a la dificultad que implica el cepillado en esta zona, como lo demuestra Quirynen y col. (10), quienes encontraron también mayor formación de placa en premolares y molares superiores.

En cuanto al estado gingival, se halló que el $45,13 \%$ presentó inflamación gingival leve (valores entre $0-1)$, el 51,62\% inflamación gingival moderada (valores entre 1,1-2) y el 3,25\% inflamación gingival severa (valores entre 2.1-3). Esto se puede comparar con lo descrito por Bustos y Oyanader (4), afirmando que la prótesis fija tiende a incrementar la inflamación gingival, ya que no permite un control óptimo de la placa bacteriana.

La retención de placa estuvo presente en el 49,6\% de casos, demostrando que los márgenes metálicos altamente pulidos y la porcelana glaseada conducen a un menor acúmulo de placa (11). Así también, Valderhaug y Karlsen (5), no encontraron diferencia significativa entre el acúmulo de placa en un diente con corona y su control (contralateral). Sin embargo, estudios como los de Anweigi y col. (6), Bustos y Oyanader (4), hallaron que las piezas pilares de prótesis parciales fijas o con una corona individual presentaban mayores índices de placa, comparadas con un diente natural.

La movilidad dentaria estuvo presente sólo el $9,7 \%$, esto puede ser debido a que las piezas con movilidad dentaria no son consideradas buenos pilares, por ello antes de realizar el tratamiento protésico, se debe eliminar el factor etiológico que causa la movilidad. Los resultados de la presente investigación son compatibles con lo sostenido por Lulic y col. (12), quienes el año 2007, luego de una revisión sistemática, concluyen que después de 10 años, la tasa estimada de prótesis parciales fijas, sin cambios en movilidad es de $93,8 \%$, resultado muy alto que corrobora las recomendaciones que con adecuados controles y mantenimiento se pueden mantener condiciones periodontales óptimas.

Con respecto al estado de las prótesis, el presente estudio encontró que el 28,3\% presentó estado bueno. Cabanilla y col. (13), encontraron como causas más frecuentes de fracaso de prótesis a los siguientes factores: caries dental, necesidad de tratamiento endodóncico, fractura, complicaciones periodontales; encontrando una supervivencia de pilares de PPF de $90 \%$ a los 4 años de cementadas, y de $75,7 \%$ a los 6 años. Así mismo, Lulic y col. (12) revisaron el impacto de la reducción severa de tejido periodontal, en la tasa de supervivencia y complicaciones de prótesis parciales fijas, encontrando una tasa de supervivencia de prótesis dentales de $96,4 \%$ después de 5 años y de $92,9 \%$ después de 10 años, concluyendo que la tasa de supervivencia de prótesis fijas en tejidos periodontales con soporte sano pero reducido, puede lograr un tratamiento protésico adecuado, exitoso y largo. 
La presente investigación encontró relación estadísticamente significativa $(\mathrm{p}<0.05)$ entre las variables: tipo de prótesis y retención de placa, encontrando un $26,3 \%$ de coronas y un $24,2 \%$ de puentes sin retención de placa, mientras que un 20,4\% de coronas y $29,2 \%$ de puentes sí presentaron esta característica, lo que podría indicar que los puentes podrían acumular mayor cantidad de placa, como también se encontró en el estudio de Valderhaugh y col. (5). Además, encontró relación significativa entre las variables tipo de prótesis y movilidad, observándose que el $9,7 \%$ de ellas, presentaban movilidad; de las cuales el 6,5\% eran pilares de puentes, coincidiendo con Valderhaugh y Karlsen (5), Lulic y col. (12), Näpänkangas y col. (7).

Las variables estado de prótesis e índice gingival unitario, también se encontraron relacionadas, obteniendo un $71,7 \%$ con mal estado de prótesis y de estas el 29,8\% presentaron gingivitis leve, 39,2\% moderada y el $2,7 \%$ severa. Estos resultados coinciden con lo descrito por Savadi y col. (14), y Nart y col. (15), acerca de la agresión que sufren los tejidos periodontales luego de ser sometidos a un tratamiento protésico fijo y más aún si esta prótesis no se encuentra en buenas condiciones. La última relación significativa encontrada entre variables fue: estado de la prótesis y retención de placa, observándose que el $43,7 \%$ de las prótesis en mal estado presentaban retención de placa.

Estudios como los de Pretzl y col. (16), Carnevale y col. (17), demostraron que los dientes usados como pilares de prótesis fijas tienen mayores posibilidades de pérdida. Por otro lado, Ikai y col. (18), reportan que no hay estudios que muestren en detalle las condiciones de las piezas pilares de prótesis fija antes del tratamiento protésico, por lo que sugieren establecer una clasificación para las piezas pilares, en la cual se refleje la predictibilidad de los resultados del tratamiento basados en las condiciones pretratamiento.

En conclusión, se observó relación entre el estado periodontal y la presencia de prótesis fija, lo cual se expresa a través en la alta prevalencia de gingivitis moderada y retención de placa en las piezas pilares de las prótesis fijas. Por tanto, es de vital importancia realizar controles rigurosos del estado periodontal antes, durante y después de cualquier tratamiento realizado en la cavidad bucal, para garantizar su éxito, funcionalidad, longevidad y por ende bienestar al paciente.

\section{Correspondencia:}

Raquel Huivín-Rodríguez

Calle Volcán Misti 124, La Molina. Lima, Perú.

Correo electrónico: rosa.huivin@upch.pe

\section{REFERENCIAS BIBLIOGRÁFICAS}

1. Pegoraro L, Do Valle A, Dos Reis C, Bonfante G, Rodríguez P, Bonachela V. Prótesis Fija. 1 ed. São Paulo: Artes médicas; 2001.

2. Rossi G, CunibertiN.Atlas de Odontología Restauradora y Periodoncia. 1 ed. Buenos Aires: Panamericana; 2004.

3. Nart J, Mor C, Baglivo M, Paniagua B, Valles C, Pascual A. Rehabilitación del paciente periodontal mediante prótesis fija dentosoportada: consideraciones prácticas y secuencias de tratamiento. Gac Dent. 2011; 228(8):6072.

4. Bustos L, Oyanader C. Condición periodontal de las prótesis fijas singulares realizadas en la clínica odontológica integral del adulto de la Universidad Mayor de Temuco. Int J Odontostomat. 2012; 6(2):195200.

5. Valderhaugh J, Karlsen K. Frequency and location of artificial crowns and fixed partial dentures constructed at a dental school. J Oral Rehabil. 1976; 3(1):75-81.

6. Anweigi L, Ziada H, Allen P. The clinical performance of hybrid bridges delivered by undergraduate dental students: a retrospective study. J Oral Rehabil. 2007; 34(4):291-6.

7. Näpänkangas R, Haikola B, Oikarinen K, Söderholm A, Remes T, Sipila K. Prevalence of single crowns and fixed partial dentures in elderly citizens in the southern and northern parts of Finland. J Oral Rehabil. 2010; 38(1):328-32.

8. Zitzmann N, Hagmann E, Weiger R. What is the prevalence of varios type of prosthetic dental restorations in Europe? Clin Oral Impl Res. 2007; 18(3):20-33.

9. Silness J. Periodontal conditions in patients treated with dental bridges. J Periodont Res. 1970; 5 (3):21924.

10. Quirynen M, Dekeyser C, Van Steenbergbe D. The influence of gingival inflammation, tooth type and timing on the rate of plaque formation. J Periodontol. 1991; 62(3):219-22.

11. Donovan T, Cho G. Predictable aesthetics with metalceramic and all-ceramic crowns: the critical importance of soft-tissue management. Periodontol 2000. 2001; 27(1):121-130.

12. Lulic M, Brägger U, Lang NP, Zwahlen M, Salvi 
GE. Ante's (1926) law revisited: a systematic review on survival rates and complications of fixed dental prostheses (FDPs) on severely reduced periodontal tissue support. Clin Oral Impl Res. 2007; 18(3):63-72.

13. Cabanilla L, Neely A, Hernandez F. The relationship between periodontal diagnosis and prognosis and the survival of prosthodontic abutments: a retrospective study. Quintessence Int. 2009; 40(10):821-31.

14. Savadi A, Rangarajan V, Savadi R, Satheesh P. Biologic perspectives in restorative treatment. J Indian Prosthodont Soc. 2011; 11(3):143-8.

15. Nart J, Mor C, Baglivo M, Paniagua B, Valles C, Pascual A. Rehabilitación del paciente periodontal mediante prótesis fija dentosoportada: consideraciones prácticas y secuencias de tratamiento. Gac Dent. 2011; 228(8):6072.

16. Pretzl B, Kaltschmitt J, Kim T, Teitmeir P, Eickholz P. Tooth loss after active periodontal therapy. II: tooth- relatedfactors. J Clin Periodontol. 2008; 35(2):175-82.

17. Carnevale G, Cairo F, Tonetti M. Long- term effects of supportive therapy in periodontal patients treated with fibre retention osseous resective surgery. II: tooth extractions during active supportive therapy. J Clin Periodontol. 2007; 34(4):342-8.

18. Ikai H, Kanno T, Kimura K. A review of clinical followup studies focusing on pretreatment conditions of abutment and clinical examination parametes. Nihon Hotetsu Shika Gakkai Zasshi. 2006; 50(2):245-55.

Recibido: 18/12/2014

Aceptado: 20/02/2015 